\section{Werben für die FMH?}

\author{
Dr. Claude Aubert, Mitglied des Zentralvorstands
}

Bevor für die FMH Werbung betrieben werden kann, muss die Frage nach der Zielgruppe geklärt werden. An welches Publikum müsste sich eine allfällige Aktion, eine Publikation oder ein Aufruf richten?

Von aussen gesehen ist die FMH eine starke Lobby, ihre Mitglieder (die meisten jedenfalls) sind sich dessen jedoch oft nicht bewusst und kritisieren das Schweigen und die Untätigkeit ihrer Verbindung. Arbeit im Schatten oder Siesta im Schatten?

Es ist ein grosser Unterschied, ob man für einen Papiertiger oder für eine Vauban'sche Festung Werbung betreibt.

Ärzte und Ärztinnen sind in der überwiegenden Mehrzahl "automatisch" Mitglieder der FMH. Diese Mitgliedschaft ist jedoch nicht gleichbedeutend mit aktiver Teilnahme. Es ist schwierig abzuschätzen, ob wir uns eher auf die verbalen Äusserungen ("sie tun nichts») stützen sollen, oder doch eher auf die Taten, speziell die Bezahlung der Mitgliederbeiträge, eine bedeutsame Geste, die als Ermunterung aufgefasst werden kann.

Gilt es, einen kämpferischen Ton anzuschlagen und persönliches Engagement zu erzwingen? Wird die Meinung der Mehrheit möglicherweise verkannt, weil die Aussagen von Worten und Taten verwechselt werden?

Die FMH besteht gemäss ihrer Statuten aus Basisorganisationen mit eigener Politik und eigenen Interessen. Ist die FMH mehr als nur die Summe ihrer Komponenten? Oder stellt sie eine Art «Drohfinger» dar, den jeder bei Bedarf erheben kann, indem er sich auf eine virtuelle Macht beruft ("Die FMH hat gesagt ..."), nur um sich dann im kritischen Moment von ihr zu distanzieren?
Soll Werbung für die FMH in erster Linie die kantonalen Ärztegesellschaften (KG), die medizinischen Fachgesellschaften (FG), den Verein der Leitenden Spitalärzte der Schweiz (VLSS) und den Verband Schweizer Assistenz- und Oberärzte/innen (VSA0) ansprechen, bevor sie Herrn oder Frau Doktor $\mathrm{Mu}-$ sterfall in ihrem Sprechzimmer erreicht?

Die Bevölkerung hat seit Jahrhunderten eine gespaltene Meinung über unseren Berufsstand. Im allgemeinen ist «unser eigener Doktor der beste, die übrigen hingegen ..."

Soll Werbung für die FMH über Medizin, das heisst, über die Medizin als Wissenschaft, informieren oder soll sie ein Loblied auf die Ärzteschaft singen, genauer gesagt, auf die Männer und Frauen, die diesen Beruf ausüben? Bedeutet Werbung, bei den anderen "verwandten oder verschwägerten" (wie es in den Todesanzeigen heisst) Berufen für unseren Berufsstand zu werben? Oder ist es ihre Aufgabe, eine von Hippokrates entworfene Lebensphilosophie zu verbreiten?

Vergessen wir die Mächtigen nicht, diejenigen, die regieren und Gesetze erlassen. Krankheitsbekämpfung ist nicht unbedingt ein uneigennütziges Anliegen. Die "politische Korrektheit" verlangt, dass der Medizin und der Gesundheit eine hohe Priorität eingeräumt wird. Es sei denn, der Zustand der öffentlichen Finanzen zwinge zu Einsparungen in diesem Sektor, damit der Säckel anderer Bittsteller gefüllt werden kann.

Bedeutet Werbung für die FMH, sich dem Hofstaat der Grossen anzuschliessen und um ihre Gunst zu buhlen, oder bedeutet sie, im Hinterhof mit den kleinen Leuten für ihre Würde zu kämpfen?

Fragen über Fragen. Fragen kann man immer stellen.

Liebe Leserin, lieber Leser, wie denken Sie darüber? 